\title{
Role of circulating endothelial progenitors in responsiveness to erythropoiesis-stimulating agents in hemodialysis patients
}

\author{
Akira Ishimitsu, Hiroshi Satonaka*, Masahito Furuichi, Yoshiki Murayama, Akihiro Tojo and Toshihiko Ishimitsu
}

\begin{abstract}
Background: Though treatment of anemia in chronic kidney disease (CKD) patients has been improved remarkably by erythropoiesis-stimulating agents (ESA), hypo-responsiveness to ESA poses a persistent problem in a subgroup of CKD patients, especially those on dialysis, with deteriorated mortality or cardiovascular risks. Pathomechanism of this condition including cardiovascular implications has not been sufficiently investigated.

Methods: Clinical parameters of 101 chronic hemodialysis patients which included those hospitalized in our university hospital were examined cross-sectionally. As a marker estimating ESA hypo-responsiveness, erythropoietin resistance index (ERI) was calculated. Numbers of circulating endothelial progenitor cells (EPC) were measured by flow cytometry. Associations among values were analyzed by methods including multiple linear regression.

Results: Majority (93\%) of the subjects were hospitalized patients with various comorbidities. ERI (18.4 [7.2-33.0] IU/ week/kg/g/dL) and ESA dose (161.6 [75.0-320.9] IU/week/kg) of all the subjects were relatively high. Factors negatively correlated with EPC included age, HD vintage, CRP, pulse rate, ESA dose and ERI, while male sex and systolic blood pressure were positively correlated. By multiple linear regression analysis, age, sex and ERI (standardized coefficient beta $-0.202, p=0.039$ ) remained as the independently predicting factors of EPC (log CD133/FIk1+EPC). Darbepoetin alpha was used in 65 patients, especially predominant when requiring higher ESA doses, but by correlation or multivariable analyses, this did not substantially modify the negative association between ERI and EPC.
\end{abstract}

Conclusions: ERI was independently associated with EPC paucity in a cohort with various comorbidities. This may suggest a link which connects ESA hypo-responsiveness to compromised cardiovascular prognosis of dialysis patients.

Keywords: Dialysis, Anemia, Erythropoietin, Erythropoiesis-stimulating agent, Endothelial progenitor cell

\section{Introduction}

Anemia is a frequent complication of chronic kidney disease (CKD) patients, for which diminished erythropoietin (EPO) production in the kidney is for the most part responsible [1]. While development and clinical use of erythropoiesis-stimulating agents (ESA) have remarkably improved treatment of CKD's anemia, it has become

*Correspondence: satonaka@dokkyomed.ac.jp Department of Nephrology and Hypertension, Dokkyo Medical University, 880 Kitakobayashi, Mibu, Tochigi 321-0293, Japan apparent that individual responses to ESA are considerably varied. This hypo-responsiveness in a subgroup of patients continues to pose a persistent problem, associated with deteriorated consequences in mortality and cardiovascular risks [2]. Though iron deficiency, inflammation, malnutrition or secondary hyperparathyroidism are among the reported frequent causes for hypo-responsiveness $[1,3]$, the whole pathological mechanism has not been adequately clarified, including its cardiovascular implications, necessitating further investigation.

Endothelial damage is observed at relatively early stages of atherosclerotic diseases [4]. It has been extensively 
shown that diagnosis and treatment of endothelial damage have important implications in cardiovascular prognosis [5]. Endothelial progenitor cells (EPC) were originally identified as circulating cells which coexpress CD34 and Flk1 (fetal liver kinase 1) which is also known as vascular endothelial growth factor receptor-2 (VEGFR-2) or kinase insert domain receptor (KDR). EPCs are thought to be produced in the bone marrow, and after being released into systemic circulation, to provide endothelial protection [6]. EPCs have been widely shown to be useful as a marker of endothelial function, and to have therapeutic utility in cardiovascular diseases, including in CKD patients [7].

Not much is known as yet on how closely ESA hyporesponsiveness is related to EPC levels, or more broadly, to endothelial function. In this cross-sectional study, we attempted to shed light on this hypothesized connection by investigating the association of EPC numbers with various clinical parameters including ERI, a marker estimating ESA hypo-responsiveness, in chronic hemodialysis (HD) patients.

\section{Methods \\ Patient recruitment, blood sample acquisition and data collection}

Any patient who underwent chronic HD treatment at Blood Purification Center, Dokkyo Medical University Hospital, from 2018 to 2020 became a study participant candidate. Study subjects were recruited from both outpatients and inpatients admitted for various treatment purposes. Those who had been treated with anti-cancer drugs or hematological transfusions, or who had undergone major surgeries during three weeks prior to the EPC measurement were excluded. We got informed consents for participation from study subjects in accordance with procedures determined by our university hospital ethics committee, which approved this study. Since we used freshly collected blood sample at the start of dialysis session for flow cytometry measurement, patient recruitment was done in so far as was made possible on the basis of measurement practicability, but without any particular biased intention in selection.

Blood sample test results, such as blood cell counts or biochemistry values, which were measured at the time of EPC sample collection, were mostly used, but in some patients, those on the closest other HD day, and within a week were adopted. Some values, which normally are not measured on a daily basis, such as total cholesterol, triglyceride or intact PTH, were adopted within a month of EPC measurement. Blood sugar levels were measured at the start of dialysis, and included non-fasting values. BNP values were adopted irrespective of the timing of dialysis session within a week from EPC measurement. Blood pressure and pulse rate at the start of dialysis session on the same day of EPC measurement were adopted. As the body weight, dry weight which was actually achieved around the time of EPC measurement was adopted. To delineate the comorbidities profiles of the study subjects which included many requiring admission for treatments, Charlson Comorbidity Index was used [8].

\section{EPC measurement by flow cytometry}

Mononuclear cell fraction was separated from $8 \mathrm{~mL}$ heparinized whole blood by gradient centrifugation using Lymphoprep (Abbot Diagnostics Technologies AS, Abbott Park, Illinois, USA). Of the $1 \mathrm{~mL}$ finally suspended mononuclear cells in PBS, $100 \mu \mathrm{L}$ was used for a set of antibodies staining. For staining, $10 \mu \mathrm{L}$ of each antibody solution was added, mixed well, and incubated for $15 \mathrm{~min}$ at room temperature, in darkness. As control, same $100 \mu \mathrm{L}$ mononuclear cells not stained was used. After the staining, sample was washed, by adding $2 \mathrm{~mL}$ PBS, and after gently mixing, cell suspension was centrifuged at $1500 \mathrm{rpm}$ for $5 \mathrm{~min}$. Washing was done twice. After the final centrifugation, sample was mixed with $300 \mu \mathrm{L}$ PBS, and the suspension was subjected to flow cytometry analysis with the use of BD FACSCalibur (BD, Franklin Lakes, NJ, USA). Antibodies used were antiHuman CD133PE (Miltenybiotec, Bergisch Gladbach, Germany), PE Mouse Anti-Human CD34 (BD, Franklin Lakes, NJ, USA) and Anti-Human VEGFR2/KDR PerCP MAb (R\&D Systems, Minneapolis, Minnesota, USA). Percentages of cells which were double positive for CD34/Flk1 or CD133/Flk1 were counted as estimates of circulating EPCs number.

\section{Erythropoietin resistance index (ERI)}

ERI was calculated as a marker estimating ESA hyporesponsiveness. Weekly ESA dose (in IU epoetin alpha or beta) per body weight kilogram was divided by hemoglobin $(\mathrm{g} / \mathrm{dl})$. ESA dose which had been administered for the longest during the three weeks up to the EPC measurement was adopted. For dose conversion between ESA types, $1 \mu \mathrm{g}$ darbepoetin alpha (DA) was regarded as equivalent to $250 \mathrm{IU}$ epoetin (either alpha or beta) and to $1.2 \mu \mathrm{g}$ epoetin beta pegol (continuous erythropoietin receptor activator [CERA]) [9].

\section{Statistics}

For comparison between two groups, $T$ test or Mann Whitney's $U$ test was used for parametric or non-parametric variables respectively. Chi-square test was used for comparison of categorical variables. Spearman's analysis was used to examine correlation between two variables, except, Pearson's method was used between $\log$ CD133/Flk+EPC and $\log$ CD34/Flk1+EPC. Multiple 
linear regression analysis with CD133/Flk+EPC as the dependent variable was performed by incorporating independent variables by forced entry mode. For comparison of ESA doses among three ESA types, one-way ANOVA followed by Bonferroni correction was performed. For all the statistical analyses, SPSS version 26 (IBM Japan, Tokyo, Japan) was used. $P$ values less than 0.05 were considered statistically significant.

\section{Results}

\section{Patient characteristics}

Patient characteristics according to high and low CD133/ Flk1+EPC groups are shown in Table 1. Majority (93\%) of the study subjects were inpatients, with relatively high median ERI (18.4 [7.2-33.0] IU/week $/ \mathrm{kg} / \mathrm{g} / \mathrm{dL})$ and ESA dose (161.6 [75.0-320.9] IU/week/ $\mathrm{kg}$ ) as a whole. In the high EPC group patients, in comparison to the low EPC group, significantly lower values were seen in age, inpatients ratio and CRP, while higher value was seen in CERA use ratio. Though there were no significant difference in total comorbidities burdens by Charlson Comorbidity Index, the low EPC group tended to include more patients in such categories as coronary artery disease (CAD), peripheral vascular disease (PVD) or congestive heart failure (CHF), but not significantly.

\section{Correlation between log CD133/Flk1+ and log CD34/ Flk1+EPCs}

Representative flow cytometry scatter plots for EPC percentage measurement are shown in Fig. 1. Raw data distributions were skewed, but after log-transformation, both CD133/Flk1+and CD34/Flk1+EPC showed normal distributions, with Kolmogorov-Smirnov statistic of 0.053 and 0.051 respectively. Histogram of log CD133/ Flk1+ EPC distribution is shown in Additional file 1: Figure S1. Log CD133/Flk1+EPC and log CD34/Flk1+EPC were closely correlated with each other $(r=0.842$, $p<0.001$, Fig. 2). We used these log-transformed values of EPCs in the following analyses.

\section{Clinical variables correlated with $\log$ CD133/Flk1+EPC and $\log$ CD34/Flk1+EPC}

We then examined factors associated with log CD133/ Flk1+EPC and log CD34/Flk1+EPC by correlation analysis (Table 2). Significant factors negatively correlated $(p<0.05)$ with either log CD133/Flk1+EPC or $\log$ CD34/Flk1+EPC were age, HD vintage, CRP, pulse rate, ESA dose, ERI and presence of PVD, while significant positively correlated factors were male sex, systolic blood pressure and CERA use. Log CD133/Flk1+EPC, in comparison to log CD34/Flk1+EPC, tended to be more closely correlated with variables such as age, HD vintage,
CRP or ERI. Overall, however, both EPCs showed similar tendencies of correlation.

\section{Multivariable association of factors with log CD133/ Flk1+EPC}

Since $\log$ CD133/Flk1+EPCs tended to be more associated with the factors above examined, we further examined this association by multiple linear regression analysis with $\log$ CD133/Flk1+EPC as the dependent variable. As independent variables, we chose, from among the above significantly correlated factors, those which are likely to be the cause rather than the result, excluding those which are mechanistically expected to have duplicity. Accordingly, we incorporated into the model age, male sex, HD vintage, CRP, systolic blood pressure, pulse rate and ERI, by forced entry mode. As the result, age, sex and ERI remained as the only independent predicting factors (Table 3). Residuals of the regression showed normal distribution with Kolmogorov-Smirnov statistic of 0.054, suggesting validity of the inference (Additional file 1: Figure $\mathrm{S} 2$ ).

\section{Similar tendencies of epoetin and DA in ERI's association with EPC}

We then examined if types of ESA influenced the above associations. ESA dose overall was closely correlated with ERI $(r=0.989, p<0.001)$, largely because ESA dose is incorporated in the calculation of ERI. Distribution of administered ESA types across the range of ESA doses were uneven, with DA predominantly used when dose requirements were higher (Fig. 3). When ESA doses among the three ESA types were compared, only difference between epoetin and DA was significant $(p<0.001)$ (Fig. 3). We tried to crudely determine, separately for each ESA type, in which direction ESA doses and ERI were associated with $\log$ CD133/Flk1+EPC by Spearman's correlation. As the result, for each of the three ESA types, ESA doses and ERI tended to be negatively associated with EPC, in the same way as the aggregate of all ESAs, though not significantly (Table 4). Also, to confirm this in multivariable context, we added use of epoetin, as opposed to DA and CERA, as one of the variables predicting log CD133/Flk1+EPC number into the same linear multiple regression model of Table 3 , and as the result, ERI's standardized coefficient beta was changed from -0.202 to -0.214 , still negatively associated and significant $(p=0.046)$ (Additional file 2: Table S1). When choice of DA, as opposed to epoetin and CERA, was instead added as one of the variables predicting log CD133/Flk1+EPC number, ERI's standardized coefficient beta was changed from -0.202 to -0.148 , no longer statistically significant $(p=0.168)$, but still association was in the negative direction (Additional file 3: Table S2). 
Table 1 Clinical characteristics of low EPC (CD133/Flk1+<0.0881\%) and high EPC (CD133/Flk1+>0.0881\%) groups

\begin{tabular}{|c|c|c|c|c|}
\hline & All patients $(n=101)$ & Low EPC group $(n=51)$ & High EPC group $(n=50)$ & $P$ value \\
\hline \multicolumn{5}{|l|}{ Demographics } \\
\hline Age, years & $66.96(10.6)$ & $69.5(8.8)$ & $64.4(11.7)$ & 0.014 \\
\hline Male sex (\%) & 69.3 & 68.6 & 70 & 0.881 \\
\hline HD vintage (years) & $5.91(2.08-12.29)$ & $6.56(3.26-11.58)$ & $5.00(1.12-13.4)$ & 0.166 \\
\hline Inpatients (\%) & 93.1 & 98.0 & 88.0 & 0.047 \\
\hline $\mathrm{BMI}\left(\mathrm{kg} / \mathrm{m}^{2}\right)$ & $22.04(3.94)$ & $22.16(4.53)$ & $21.93(3.27)$ & 0.769 \\
\hline \multicolumn{5}{|l|}{ Clinical measurements } \\
\hline Albumin (g/dL) & $2.98(0.49)$ & $2.90(0.49)$ & $3.06(0.48)$ & 0.094 \\
\hline BUN (mg/dL) & $43.8(16.0)$ & $43.8(15.1)$ & $43.7(16.9)$ & 0.979 \\
\hline Creatinine (mg/dL) & $8.19(2.02)$ & $7.93(1.91)$ & $8.44(2.12)$ & 0.216 \\
\hline adjusted Ca (mg/dL) & $9.58(0.79)$ & $9.65(0.72)$ & $9.49(0.84)$ & 0.306 \\
\hline Phosphate (mg/dL) & $4.22(1.45)$ & $4.13(1.32)$ & $4.32(1.57)$ & 0.516 \\
\hline $\mathrm{CRP}(\mathrm{mg} / \mathrm{dL})$ & $0.86(0.21-3.11)$ & $1.44(0.27-4.55)$ & $0.63(0.15-1.69)$ & 0.017 \\
\hline $\mathrm{WBC}\left(/ \mathrm{mm}^{3}\right)$ & $6100(4900-7150)$ & $6200(4900-7000)$ & $6050(4300-7225)$ & 0.649 \\
\hline Hemoglobin (g/dL) & $9.97(1.59)$ & $9.95(1.71)$ & $9.99(1.47)$ & 0.902 \\
\hline Serum iron $(\mu \mathrm{g} / \mathrm{dL})$ & $40.0(27.5-63.5)$ & $39.0(26.0-64.0)$ & $40.5(30.0-57.0)$ & 0.831 \\
\hline TSAT (\%) & $21.7(12.7)$ & $21.5(12.9)$ & $21.8(12.6)$ & 0.909 \\
\hline Ferritin (ng/mL) & $118.8(53.4-241.2)$ & $121.4(62.7-245.4)$ & $117.3(43.1-243.4)$ & 0.545 \\
\hline Glucose (mg/dL) & $144.4(49.0)$ & $142.2(34.5)$ & $146.7(60.6)$ & 0.649 \\
\hline Total cholesterol (mg/dL) & $150.9(43.3)$ & $148.0(43.4)$ & $153.8(43.4)$ & 0.503 \\
\hline Triglyceride (mg/dL) & $100.5(45.4)$ & $102.2(49.1)$ & $98.8(41.8)$ & 0.709 \\
\hline Intact PTH (pg/mL) & $117.6(63.2-207.6)$ & $113.8(60.5-178.8)$ & $124.0(63.8-217.0)$ & 0.386 \\
\hline BNP $(p g / m L)$ & $338.7(134.5-881.8)$ & $315.2(151.0-1096)$ & $350.1(99.6-815.1)$ & 0.325 \\
\hline $\mathrm{SBP}(\mathrm{mmHg})$ & $145.0(26.9)$ & $140.5(24.4)$ & $149.6(28.8)$ & 0.088 \\
\hline $\mathrm{DBP}(\mathrm{mmHg})$ & $75.0(14.79)$ & $72.3(13.9)$ & $77.7(15.3)$ & 0.068 \\
\hline Pulse rate (/min) & $75.5(13.5)$ & $77.3(15.0)$ & $73.7(11.6)$ & 0.177 \\
\hline CD133/Flk1 + EPC (\%) & $0.0880(0.0370-0.1615)$ & $0.0393(0.0264-0.0613)$ & $0.1615(0.1168-0.3926)$ & $<0.001$ \\
\hline CD34/FIk1 + EPC (\%) & $0.0723(0.0381-0.1502)$ & $0.0397(0.0175-0.0687)$ & $0.1303(0.0741-0.3755)$ & $<0.001$ \\
\hline ERI (IU/week/kg/g/dL) & $18.4(7.2-33.0)$ & $20.4(8.0-46.7)$ & $15.6(6.6-29.0)$ & 0.240 \\
\hline \multicolumn{5}{|l|}{ Comorbidities } \\
\hline Charlson index & $7.0(6.0-9.0)$ & $8.0(6.0-9.0)$ & $7.0(6.0-9.0)$ & 0.210 \\
\hline CAD (\%) & 23.8 & 29.4 & 18.0 & 0.178 \\
\hline CVD (\%) & 17.8 & 19.6 & 16.0 & 0.636 \\
\hline PVD (\%) & 23.8 & 29.4 & 18.0 & 0.178 \\
\hline CHF (\%) & 27.7 & 33.3 & 22.0 & 0.203 \\
\hline Dementia (\%) & 2.0 & 3.9 & 0 & 0.157 \\
\hline DM (\%) & 49.5 & 52.9 & 46.0 & 0.485 \\
\hline Malignancy (\%) & 23.8 & 25.5 & 22.0 & 0.680 \\
\hline \multicolumn{5}{|l|}{ Treatments } \\
\hline ESA dose (IU/week/kg) & $161.6(75.0-320.9)$ & $179.0(80.4-444.4)$ & $150.5(71.0-285.1)$ & \\
\hline Epoetin (\%) & 30.7 & 29.4 & 32.0 & 0.778 \\
\hline DA (\%) & 64.4 & 70.6 & 58.0 & 0.187 \\
\hline CERA (\%) & 5.0 & 0 & 10.0 & 0.021 \\
\hline Iron agent (\%) & 36.6 & 43.1 & 30.0 & 0.171 \\
\hline Vitamin D (\%) & 68.3 & 66.7 & 70.0 & 0.719 \\
\hline CaSR agonist (\%) & 32.7 & 35.3 & 30.0 & 0.571 \\
\hline
\end{tabular}

Continuous variables are expressed as mean (SD) or median (interquartile range). Categorical variables are expressed as percentages. $P$ values in bold indicate statistical significance

BMI, body mass index; BUN, blood urea nitrogen; $\mathrm{Ca}$, calcium; CRP, C-reactive protein; WBC, white blood cell; TSAT, transferrin saturation; PTH, parathyroid hormone; BNP, brain natriuretic peptide; SBP, systolic blood pressure; DBP, diastolic blood pressure; CAD, coronary artery disease; CVD, cerebral vascular disease; PVD, peripheral vascular disease; CHF, congestive heart failure; DM, diabetes mellitus; DA, darbepoetin alpha; CERA, continuous erythropoietin receptor activator; CaSR, calciumsensing receptor 

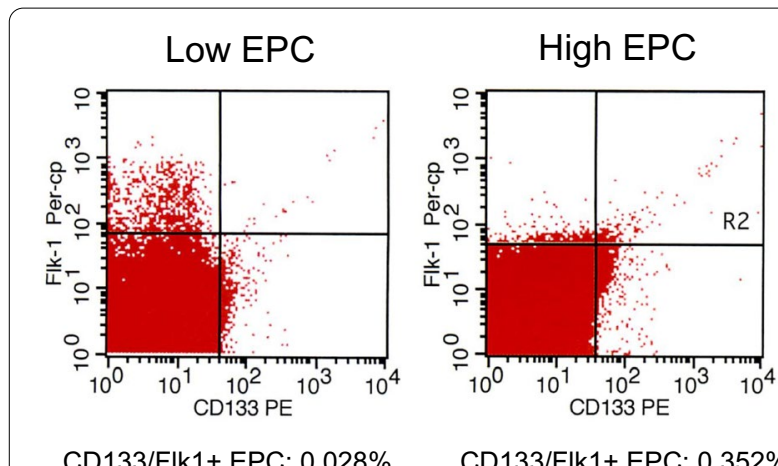

CD133/Flk1+ EPC: $0.028 \%$

Fig. 1 Representative images of flow cytometry scatter plots showing high EPC and low EPC numbers. Flow cytometry scatter plots showing high (0.028\%) and low (0.352\%) numbers of EPC. EPC numbers were counted as percentages of cells which were double positive for CD133 and Flk1, in circulating mononuclear cells

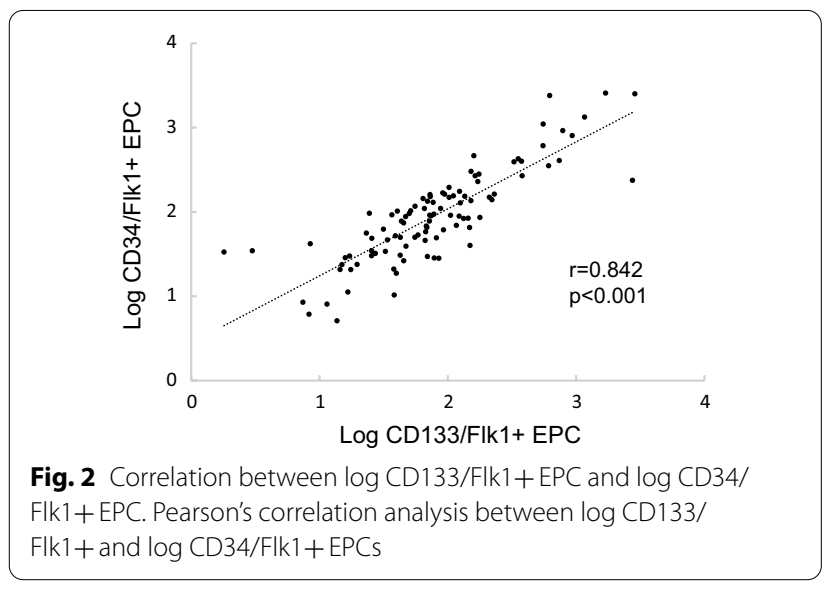

These results suggested that though whole ERIs' negative association with EPC may have been more influenced by DA's larger share of contribution especially at higher doses, both epoetin and DA seemed to have similar tendencies in these associations. Since CERA was used in a very small number of patients $(n=5)$, its association may be affected by coincidence, and was regarded not worth examining.

\section{No involvement of iron status in EPC levels or EPCs' association with ERI}

As shown in Table 2, values reflecting iron status, namely serum iron, TSAT and ferritin, were not correlated with EPC levels. We further compared subgroups of subjects with or without iron deficiency. Iron deficiency was defined as TSAT $<20 \%$ and ferritin $<100 \mathrm{ng} / \mathrm{ml}$, according to 2015 Japanese Society for Dialysis Therapy: Guidelines for Renal Anemia in Chronic Kidney Disease [10].
As shown in Fig. 4, distributions of subjects according to $\log$ CD133/Flk1+EPC levels in subgroup with iron deficiency $(n=24)$ and subgroup without iron deficiency $(n=77)$ were similar by Mann-Whitney $U$ test $(p=0.473)$. As shown in Additional file 4: Table S3, tendencies of correlations between log CD133/Flk1+EPC with ERI were similarly in negative direction in these subgroups.

\section{Discussion}

In this analysis of a relatively small-size cohort of dialysis patients, age, sex and ERI were the independent predicting factors of circulating EPC number. Though involvement of EPO or ESA in endothelial function has been previously demonstrated, our present study may be one of the few, if any, to have shown the association between a marker of ESA hypo-responsiveness and decreased EPC.

\section{Comparison of ERI and ESA dose in terms of overall associations with variables}

ESA hypo-responsiveness would be, as a concept, an index reflecting degree of body's inability to react to secreted EPO, just like insulin resistance in diabetes. But in actual clinical situations, increased EPO dose itself in hypo-responsive patients may also further exacerbate the condition. ERI has been generally accepted as a marker which is static in nature but capable of estimating dynamic ESA hypo-responsiveness. Though prognostic utility of ERI is extensively shown [11], it is by definition correlated with ESA dose itself. It may be in reality difficult to distinguish effects of ESA dose from ESA hyporesponsiveness. In our present study, when correlations with various clinical parameters were compared between ESA dose and ERI, patterns were similar, almost identical, though there were slight differences in association levels with variables like albumin (negative correlation), or CRP (positive correlation) (Additional file 5: Table S4). With such variables, ERI tended to be associated slightly more strongly, than ESA dose was. This may be because systemic conditions like inflammation or malnutrition, in addition to ESA dose itself, contributed to hypo-responsiveness to ESA.

\section{ERI of the study subjects in relation to definition of ESA hypo-responsiveness}

"ESA hypo-responsiveness" is a term generally used for patients who require relatively high doses of ESAs to increase or maintain hemoglobin levels. Currently there is no established quantitative definition of ESA hypo-responsiveness. Kidney Disease: Improving Global Outcomes (KDIGO) guidelines define those patients 
Table 2 Variables correlated with log CD133/Flk1+and log CD34/Flk1+EPCs

\begin{tabular}{|c|c|c|c|c|}
\hline & \multicolumn{2}{|c|}{$\underline{\log C D 133 / F I k 1+E P C}$} & \multicolumn{2}{|c|}{$\log$ CD34/Flk1+EPC } \\
\hline & $r$ & $p$ & $r$ & $p$ \\
\hline Age & -0.215 & 0.031 & -0.178 & 0.075 \\
\hline Male sex & 0.134 & 0.182 & 0.209 & 0.036 \\
\hline HD vintage & -0.232 & 0.019 & -0.215 & 0.031 \\
\hline Inpatients & -0.274 & 0.006 & -0.181 & 0.071 \\
\hline $\mathrm{BMI}$ & 0.070 & 0.486 & 0.062 & 0.539 \\
\hline Albumin & 0.194 & 0.052 & 0.105 & 0.297 \\
\hline BUN & 0.062 & 0.540 & -0.022 & 0.830 \\
\hline Creatinine & 0.179 & 0.073 & 0.114 & 0.256 \\
\hline adjusted Ca & -0.129 & 0.198 & -0.131 & 0.190 \\
\hline Phosphate & 0.144 & 0.151 & 0.133 & 0.185 \\
\hline CRP & -0.294 & 0.003 & -0.236 & 0.017 \\
\hline WBC & -0.007 & 0.947 & -0.031 & 0.759 \\
\hline Hemoglobin & 0.082 & 0.418 & 0.011 & 0.915 \\
\hline Serum iron & -0.018 & 0.858 & -0.053 & 0.601 \\
\hline TSAT & 0.014 & 0.890 & -0.005 & 0.960 \\
\hline Ferritin & -0.094 & 0.350 & -0.071 & 0.482 \\
\hline Glucose & -0.077 & 0.445 & -0.081 & 0.420 \\
\hline Total cholesterol & 0.046 & 0.650 & -0.041 & 0.686 \\
\hline Triglyceride & -0.065 & 0.516 & -0.065 & 0.520 \\
\hline Intact PTH & 0.105 & 0.298 & 0.050 & 0.620 \\
\hline BNP & -0.121 & 0.228 & -0.068 & 0.501 \\
\hline SBP & 0.172 & 0.085 & 0.203 & 0.042 \\
\hline DBP & 0.189 & 0.058 & 0.192 & 0.055 \\
\hline Pulse rate & -0.184 & 0.065 & -0.206 & 0.039 \\
\hline ERI & -0.207 & 0.037 & -0.139 & 0.167 \\
\hline Charlson index & -0.094 & 0.352 & -0.042 & 0.676 \\
\hline CAD & -0.11 & 0.273 & -0.115 & 0.253 \\
\hline CVD & -0.055 & 0.585 & -0.067 & 0.508 \\
\hline PVD & -0.202 & 0.043 & -0.146 & 0.145 \\
\hline $\mathrm{CHF}$ & -0.147 & 0.142 & -0.178 & 0.076 \\
\hline Dementia & -0.158 & 0.114 & -0.171 & 0.088 \\
\hline DM & -0.084 & 0.402 & -0.105 & 0.295 \\
\hline Malignancy & 0 & 1.000 & 0.079 & 0.432 \\
\hline ESA dose & -0.206 & 0.039 & -0.138 & 0.168 \\
\hline Epoetin use & 0.023 & 0.821 & 0.039 & 0.698 \\
\hline DA use & -0.165 & 0.099 & -0.179 & 0.074 \\
\hline CERA use & 0.316 & 0.001 & 0.312 & 0.002 \\
\hline Iron agent use & -0.063 & 0.529 & 0.053 & 0.600 \\
\hline Vitamin D use & -0.053 & 0.602 & -0.144 & 0.151 \\
\hline CaSR agonist use & -0.115 & 0.252 & -0.11 & 0.273 \\
\hline
\end{tabular}

Correlations with $\log$ CD133/Flk1 + and CD34/FIk1+EPC were examined by Spearman's method. $P$ values in bold indicate statistical significance

$\mathrm{BMI}$, body mass index; BUN, blood urea nitrogen; $\mathrm{Ca}$, calcium; CRP, C-reactive protein; WBC, white blood cell; TSAT, transferrin saturation; PTH, parathyroid hormone; BNP, brain natriuretic peptide; SBP, systolic blood pressure; DBP, diastolic blood pressure; $C A D$, coronary artery disease; $C V D$, cerebral vascular disease; $P V D$, peripheral vascular disease; $C H F$, congestive heart failure; $D M$, diabetes mellitus; DA, darbepoetin alpha; CERA, continuous erythropoietin receptor activator; CaSR, calcium-sensing receptor
Table 3 Variables predicting log CD133/Flk1+EPC in multiple linear regression analysis

\begin{tabular}{|c|c|c|c|c|c|}
\hline \multirow[t]{2}{*}{$\begin{array}{l}\text { Independent } \\
\text { variables }\end{array}$} & \multicolumn{2}{|c|}{$\begin{array}{l}\text { Unstandardized } \\
\text { coefficients }\end{array}$} & \multirow{2}{*}{$\begin{array}{l}\text { Standardized } \\
\text { coefficients } \\
\text { Beta }\end{array}$} & \multirow[t]{2}{*}{$t$} & \multirow[t]{2}{*}{$P$ value } \\
\hline & $B$ & SE & & & \\
\hline Age & -0.012 & 0.005 & -0.244 & -2.416 & 0.018 \\
\hline ERI & -0.004 & 0.002 & -0.202 & -2.096 & 0.039 \\
\hline Male sex & 0.217 & 0.108 & 0.186 & 2.012 & 0.047 \\
\hline CRP & -0.026 & 0.018 & -0.139 & -1.433 & 0.155 \\
\hline SBP & 0.003 & 0.002 & 0.132 & 1.391 & 0.167 \\
\hline HD vintage & -0.009 & 0.007 & -0.129 & -1.343 & 0.182 \\
\hline Pulse rate & -0.005 & 0.004 & -0.128 & -1.255 & 0.213 \\
\hline
\end{tabular}

Age, male sex, $\mathrm{HD}$ vintage, CRP, SBP, pulse rate and ERI were incorporated as predicting variables into the model by forced entry mode, with log CD133/ Flk1+EPC as dependent variable. $P$ values in bold indicate statistical significance CRP, C-reactive protein; SBP, systolic blood pressure

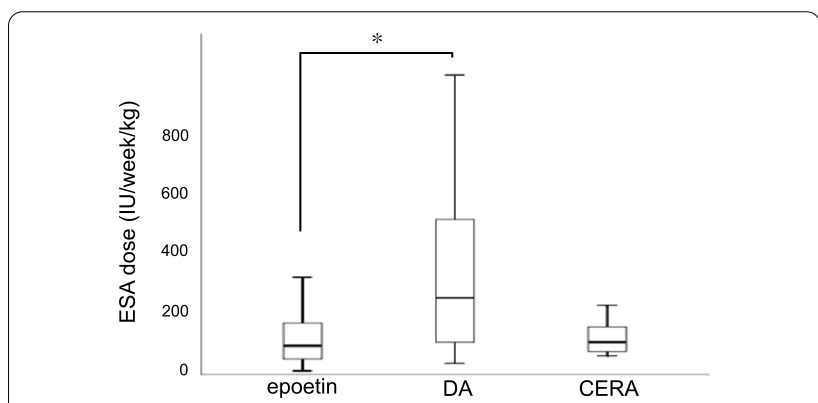

Fig. 3 Distribution of ESA doses according to each ESA type. Median and interquartile ranges of ESA doses according to ESA type are shown. One microgram darbepoetin alpha (DA) was regarded as equivalent to $250 \mathrm{IU}$ epoetin and to $1.2 \mu \mathrm{g}$ epoetin beta pegol (continuous erythropoietin receptor activator [CERA]). ${ }^{*} p<0.001$

Table 4 Correlations of ESA dose and ERI with log CD133/ Flk1+EPC, according to ESA type

\begin{tabular}{llllll}
\hline & \multicolumn{2}{l}{$\begin{array}{l}\text { ESA dose versus log } \\
\text { CD133/FIk1+EPC }\end{array}$} & & \multicolumn{2}{l}{$\begin{array}{l}\text { ERI versus log } \\
\text { CD133/FIk1+EPC }\end{array}$} \\
\cline { 2 - 3 } & $\boldsymbol{r}$ & $\boldsymbol{p}$ & & $\boldsymbol{r}$ & $\boldsymbol{p}$ \\
\hline All subjects $(n=101)$ & -0.206 & $\mathbf{0 . 0 3 9}$ & & -0.207 & $\mathbf{0 . 0 3 7}$ \\
Epoetin $(n=31)$ & -0.176 & 0.343 & & -0.157 & 0.398 \\
DA $(n=65)$ & -0.194 & 0.122 & & -0.213 & 0.088 \\
CERA $(n=5)$ & -0.300 & 0.624 & & -0.600 & 0.285
\end{tabular}

Correlations of ESA dose and ERI with log CD133/FIk1+EPC were analyzed by Spearman's method. $P$ values in bold indicate statistical significance

$\mathrm{DA}$, darbepoetin alpha; CERA, continuous erythropoietin receptor activator 


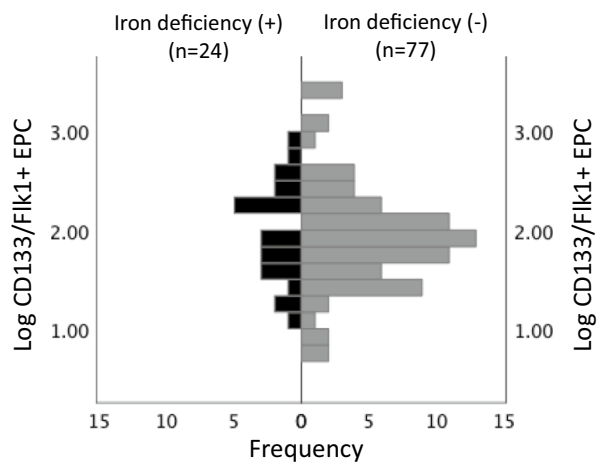

Fig. 4 Distribution of subjects according to $\log$ CD133/Flk1+EPC in iron status subgroups. Distributions of subjects according to log CD133/Flk1+EPC levels in subgroup with iron deficiency $(n=24)$ and subgroup without iron deficiency $(n=77)$ were similar by MannWhitney $U$ test $(p=0.473)$. Iron deficiency status was defined as TSAT $<20 \%$ and ferritin $<100 \mathrm{ng} / \mathrm{ml}$

as hypo-responsive whose hemoglobin levels do not increase within the first month of ESA treatment even with appropriate weight-based dosing [12]. In a clinical practice population-based study, approximately $20 \%$ of incident ESA users, irrespective of ESA type including originator or biosimilar, were non-responders defined as with less than zero increase in hemoglobin level in comparison to the baseline during the first three months of treatment [13]. With respect to significance of ERI, which is a static, thus estimating marker of dynamic ESA hyporesponsiveness, a study which observed HD patients for a median of 4.6 years suggested baseline ERI of 11.31 as a prognostic hypo-responsiveness threshold predicting combined cardiovascular events and mortality outcome [14]. This may indicate that the median ERI of 18.4 (7.233.0) in our present cohort was relatively high, with the majority in the suggested hypo-responsive category.

\section{Endothelial pathophysiology and ESA hypo-responsiveness}

The exact mechanism linking ESA hypo-responsiveness to fewer EPCs cannot be determined from our cross-sectional study. Possibility of direct effects of EPO on EPCs has been shown previously, some studies suggesting that EPO may be beneficial by promoting EPC release. EPO has been shown to mobilize EPC release from the bone marrow and to promote neovascularization in mice [15]. Four-week DA administration to stable coronary artery disease patients increased CD34/CD133+EPCs and improved flow-mediated dilatation (FMD) findings, a marker of endothelial function [16]. Evidence is limited in CKD patients, but one study has reported administration of epoetin, but not of DA, to stage 5 patients either on HD or not yet, increased circulating EPC numbers [17].
More broadly, EPO's role in endothelial function in general is ambiguous. It has been showed that EPO directly improved FMD findings in diabetic rats [18], while there was no correlation between EPO concentrations and endothelial function in healthy humans [19].

In contrast to the above potentially beneficial effects of EPO on endothelium, there is accumulated evidence that EPO causes hypertension, where proposed mechanism are wide-ranging, including deranged NO regulation, endothelins, sympathoadrenal system, or renin-angiotensin system [20]. Overall, judging from numerous mutually conflicting reports, EPO may exert variable cardiovascular effects, possibly depending on clinical contexts. Though it is only speculative, the role of EPO may be altered depending on the pro-inflammatory conditions of CKD, as in our study subjects, with its harmful aspects against the endothelium or cardiovascular organs being accentuated.

\section{Possible role of bone marrow}

Otherwise, concerning bone marrow's involvement, secondary hyperparathyroidism, frequently a complication of CKD and an incitement for ESA resistance, is reported to deleteriously affect bone marrow hematopoiesis [21]. Though importance of PTH was not evident in our study, various likewise adverse mediators under the systemic condition of ESA resistance may affect EPC production in the bone marrow. Recently, it was reported that administration of anti-BMP6 (bone morphogenic protein 6) antibody ameliorated mouse models of CKD's anemia, whereby it suppressed hepcidin and increased ferroportin expression in erythroid precursor cells, accelerating erythroid output [22]. Since bone marrow is also involved in the modulation of EPC abundance, our findings may further highlight a potentially pivotal role of bone marrow in ESA hypo-responsiveness, explaining its cardiovascular implications.

\section{ESA types in associations between hypo-responsiveness and EPC}

With respect to differences in mortality risks depending on ESA type, a recent study examined effects of "shortacting" epoetin and "long-acting" DA on mortality in close to 200,000 HD patients, in which DA administration, in comparison to epoetin, deteriorated two-year mortality, including cardiovascular deaths [23]. In our present study, we analyzed possible differences between ESA types in the modulation of ERI's associations with EPC. Since DA, within its range of approved doses, can be used at more potent doses (up to $180 \mu \mathrm{g}$ per week) than equivalent epoetin doses, it was more likely to be chosen in patients requiring high doses. This made the direct comparison between ESA types difficult. 
However, after dose conversion by generally accepted ratios between ESAs, no substantial difference in effects was seen between epoetin and DA, both of which were associated with CD133/Flk1 + EPCs in the same negative direction as the aggregate of all three ESAs.

\section{EPC subtypes and associations with ESA hypo-responsiveness}

Among several definitions of EPCs, we measured CD133/ Flk1+and CD34/Flk1+cells. They were closely correlated with each other, and demonstrated similar patterns of association with various clinical parameters. However, CD133/Flk1+EPCs tended to be more strongly associated with a number of factors, especially those related to conditions of inflammation or malnutrition, such as age, HD vintage, albumin, CRP and ERI. ERI was significantly correlated with CD133/Flk1+EPC, but not with CD34/ Flk1+EPC. CD133Flk1+EPC is regarded as an immature, younger type, which may have distinct effects depending on disease conditions [24]. In rheumatoid arthritis patients, coronary calcification was more associated with CD133/Flk1+EPC than with CD34/Flk1+EPC, where it was speculated to be due to susceptibility of immature CD133/Flk+EPC to cytokines alteration caused by this disease [25]. Similar mechanism may play a role in HD patients, who are in general in states of chronic inflammation, and further investigation is warranted for elucidation.

\section{Implications for treatment}

This study may have implications for the treatment of CKD's anemia. Since current clinical guidelines in general recommend target $\mathrm{Hb}$ levels in $\mathrm{HD}$ patients not $<10-11.5 \mathrm{~g} / \mathrm{dL}$ [12], ESA doses are likely to be increased toward that level in cases of hypo-responsiveness, leading to high ERI values. In such conditions, additional increases ESA doses may no longer be much effective in improving anemia, and may exert detrimental effects, including cardiovascular effects, possibly as a result of ESA hypo-responsiveness. In such cases, as is suggested in clinical guidelines [12], balanced, restrained doses of ESA may be appropriate, even if only hemoglobin levels lower than the recommended targets will be achieved.

Alternatively, treatments to improve systemic conditions which promote hypo-responsiveness to ESA, such as chronic inflammation, malnutrition or frailty may be beneficial. In recent years, oral hypoxia-inducible factor prolyl hydroxylase inhibitors (HIF-PHIs), a new class of drugs which increase endogenous erythropoietin production, have become available for clinical use. HIF-PHIs have been shown to improve anemia not inferiorly in comparison to ESAs, and are presumed to increase EPO to relatively mild levels which may be closer to physiological condition [26]. HIF-PHIs have also been shown to improve iron utilization by mechanisms including down-regulation of hepcidin [27]. These effects of HIF-PHIs may be beneficial for the correction of conditions of ESA hypo-responsiveness, which may be accompanied by disordered endothelial physiology, as suggested in our present study, warranting further investigation.

\section{EPC in HD patients according to sex}

In our multivariable analysis, positive independent association of male sex with log CD133/Flk1+EPC was observed. However, when log CD133/Flk1+EPC levels were directly compared between the male and female subjects, males overall showed slightly higher values, but there was no significant statistical difference (data not shown). A recent study has shown that in women without cardiovascular diseases, reduced circulating progenitor cells, including those expressing CD34 or CD133, were observed in comparison to men [28]. This study suggested that EPC scarcity, rather than estrogen deficiency, may be responsible for the fact that lower cardiovascular incidence observed in reproductive-age women, disappears in postmenopausal or diabetic individuals, for whom hormone replacement therapy is not sufficiently effective. Though our finding in respect to EPC in female HD patients may be in agreement with such observations, it may be difficult to draw definitive conclusion since sex is a categorical variable, and especially with the relatively small sample size, other confounding factors may have influenced.

\section{Limitations}

There are several limitations to this study. First, due to the relatively small sample size, the possibility may not be ruled out that the results were due to coincidence, though the results of multivariate analysis, reached after taking relevant clinical parameters into account, may well be reasonable. Second, since the study subjects were predominantly inpatients, some with relatively heavy comorbidities burdens and requiring high ESA doses, the findings may not necessarily be applicable to the conditions of more general, stable HD patients. Nonetheless, with an increasing proportion of patients undergoing long-term HD treatments in recent years, the conditions of this study's subjects may be one aspect of the general tendency, which could only be elucidated for the very reason of this particular character of our cohort. Third, because of the cross-sectional design of this study, causality cannot be conclusively determined. Decreased EPC number may not be the result of ESA hypo-responsiveness, and some other underlying condition may be more dominant, though 
with the former as the presumed result, our multiple regression analysis results suggested the latter is likely to be a significant independent predictor among various relevant factors.

In conclusion, this study has revealed independent association of ERI, a marker estimating ESA hyporesponsiveness, with EPC paucity in hemodialysis patients, suggesting the possibility of a close link between ESA hypo-responsiveness and endothelial dysfunction, which seems to have not been demonstrated so far. This finding may be useful for devising appropriate treatment of CKD's anemia, especially taking into account cardiovascular implications.

\section{Supplementary Information}

The online version contains supplementary material available at https://doi. org/10.1186/s41100-021-00374-4.

Additional file 1: Figure S1. Normal distribution of log-transformed CD133/Flk1 + EPC numbers in percentages. CD133/FIk1+EPC numbers by percentages were multiplied by 1,000 and log-transformed with base of 10. Though distribution of EPC number in percentages was skewed, after log-transformation, data were normally distributed. Figure S2. Normal distribution of standardized residuals in multiple linear regression analysis for variables predicting CD133/Flk+1 EPC. Histogram distribution of standardized residuals in the multiple linear regression analysis of Table 3 , which analyzed independent variables predicting log CD133/FIk1+EPC. Kolmogorov-Smirnov statistic: 0.054.

Additional file 2: Table S1. Multiple linear regression analysis with epoetin use added as independent variable predicting log CD133/Flk1+EPC. Age, male sex, HD vintage, CRP, SBP, pulse rate, ERI and Epoetin use were incorporated as predicting variables into the model by forced entry mode, with log CD133/FIk1 + EPC as dependent variable. CRP, C-reactive protein; SBP, systolic blood pressure.

Additional file 3: Table S2. Multiple linear regression analysis with DA use added as independent variable predicting log CD133/Flk1+ EPC. Age, male sex, HD vintage, CRP, SBP, pulse rate, ERI and DA use were incorporated as predicting variables into the model by forced entry mode, with $\log$ CD133/FIk1 + EPC as dependent variable. CRP, C-reactive protein; SBP, systolic blood pressure.

Additional file 4: Table S3. Spearman's correlations between log CD133/ Flk1+EPC and ERI, according to iron deficiency status. Iron deficiency (TSAT $<20 \%$ and ferritin $<100 \mathrm{ng} / \mathrm{ml}$ ) was defined according to 2015 Japanese Society for Dialysis Therapy: Guidelines for Renal Anemia in Chronic Kidney Disease.

Additional file 5: Table S4. Variables correlated with ESA dose and ERI. Correlations with ESA dose and ERI were examined by Spearman's method. BMI, body mass index; BUN, blood urea nitrogen; Ca, calcium; CRP, C-reactive protein; WBC, white blood cell; TSAT, transferrin saturation; PTH, parathyroid hormone; BNP, brain natriuretic peptide; SBP, systolic blood pressure; DBP, diastolic blood pressure; CAD, coronary artery disease; CVD, cerebral vascular disease; PVD, peripheral vascular disease; CHF, congestive heart failure; DM, diabetes mellitus; DA, darbepoetin alpha; CERA, continuous erythropoietin receptor activator; CaSR, calcium-sensing receptor.

\section{Acknowledgements}

We are grateful to Yasuko Nonaka (Center for Research Collaboration and Support, Dokkyo Medical University) for technical assistance with flow cytometry, and to Kyoko Tabei, Kazumi Akimoto and Atsuko Makino (Center for Research Collaboration and Support, Dokkyo Medical University) for technical assistance with mononuclear cell isolation.

\section{Authors' contributions}

Al wrote the paper with intellectual input from all authors. Conception and research design: $\mathrm{Al}, \mathrm{HS}, \mathrm{Tl}$; data acquisition: $\mathrm{Al}, \mathrm{HS}, \mathrm{MF}, \mathrm{YM}$; data analysis and interpretation: Al, HS; statistical analysis: Al, HS; supervision and mentorship: AT, TI. All authors have approved the manuscript.

Funding

The authors received no funding for this work.

\section{Availability of data and materials}

The dataset generated and/or analyzed during the current study are available from corresponding author on reasonable request.

\section{Declarations}

Ethics approval and consent to participate

This study protocol complied with the Declaration of Helsinki and was approved by the ethics committee of Dokkyo Medical University (Acceptance No. R-20-1 J). All the study subjects received an explanation of the procedures and possible risks of this study and gave their informed consent to participate.

\section{Consent for publication}

Not applicable.

\section{Competing interests}

The authors declare that they have no competing interest.

Received: 2 April 2021 Accepted: 30 September 2021

Published online: 14 October 2021

\section{References}

1. Babitt JL, Lin HY. Mechanisms of anemia in CKD. J Am Soc Nephrol. 2012;23(10):1631-4.

2. Akizawa T, Saito A, Gejyo F, Suzuki M, Nishizawa Y, Tomino Y, Tsubakihara Y, Akiba T, Hirakata H, Watanabe Y, Kawanishi H, Bessho M, Udagawa Y, Aoki K, Uemura Y, Ohashi Y, JET Study Group. Low hemoglobin levels and hypo-responsiveness to erythropoiesis-stimulating agent associated with poor survival in incident Japanese hemodialysis patients. Ther Apher Dial. 2014;18(5):404-13.

3. Tanaka M, Komaba H, Fukagawa M. Emerging association between parathyroid hormone and anemia in hemodialysis patients. Ther Apher Dial. 2018;22(3):242-5.

4. Ross R. Atherosclerosis: an inflammatory disease. N Engl J Med. 1999;340(2):115-26.

5. Davignon J, Ganz P. Role of endothelial dysfunction in atherosclerosis. Circulation. 2004;109(23 Suppl 1):227-32.

6. Asahara T, Murohara T, Sullivan A, Silver M, van der Zee R, Li T, Witzenbichler B, Schatteman G, Isner JM. Isolation of putative progenitor endothelial cells for angiogenesis. Science. 1997;275(5302):964-7.

7. Bahlmann FH, Speer T, Fliser D. Endothelial progenitor cells in chronic kidney disease. Nephrol Dial Transplant. 2010;25(2):341-6.

8. Charlson M, Szatrowski TP, Peterson J, Gold J. Validation of a combined comorbidity index. J Clin Epidemiol. 1994;47(11):1245-51.

9. Hasegawa T, Zhao J, Fuller DS, Bieber B, Zee J, Morgenstern H, Hanafusa N, Nangaku M. Erythropoietin hyporesponsiveness in dialysis patients: possible role of statins. Am J Nephrol. 2017;46(1):11-7.

10. Yamamoto H, Nishi S, Tomo T, Masakane I, Saito K, Nangaku M, Hattori M, Suzuki T, Morita S, Ashida A, Ito Y, Kuragano T, Komatsu Y, Sakai K, Tsubakihara Y, Tsuruya K, Hayashi T, Hirakata H, Honda H. 2015 Japanese Society for Dialysis Therapy: guidelines for renal anemia in chronic kidney disease. Renal Replace Ther. 2017:3:36.

11. Panichi V, Rosati A, Bigazzi R, Paoletti S, Mantuano E, Beati S, Marchetti V, Bernabini G, Grazi G, Rizza GM, Migliori M, Giusti R, Lippi A, Casani A, Barsotti G, Tetta C, RISCAVID Study Group. Anaemia and resistance to erythropoiesis-stimulating agents as prognostic factors in haemodialysis patients: results from the RISCAVID study. Nephrol Dial Transplant. 2011;26(8):2641-8. 
12. International Society of Nephrology. KDIGO clinical practice guideline for anemia in chronic kidney disease. Kidney Int Suppl. 2012;2(4):299-310.

13. Ingrasciotta $Y$, Giorgianni F, Marcianò I, Bolcato J, Pirolo R, Chinellato A, lentile V, Santoro D, Genazzani AA, Alibrandi A, Fontana A, Caputi AP, Trifirò G. Comparative effectiveness of biosimilar, reference product and other erythropoiesis-stimulating agents (ESAs) still covered by patent in chronic kidney disease and cancer patients: an Italian population-based study. PLoS ONE. 2016;11(5):e0155805.

14. Tanaka K, Fujiwara M, Saito H, Iwasaki T, Oda A, Watanabe S, Kanno M, Kimura H, Tani Y, Asai J, Suzuki H, Sato K, Kazama JJ. Hyporesponsiveness to long-acting erythropoiesis-stimulating agent is related to the risk of cardiovascular disease and death in Japanese patients on chronic hemodialysis: observational cohort study. Renal Replace Ther. 2021;7:13.

15. Heeschen C, Aicher A, Lehmann R, Fichtlscherer S, Vasa M, Urbich C, Mildner-Rihm C, Martin H, Zeiher AM, Dimmeler S. Erythropoietin is a potent physiologic stimulus for endothelial progenitor cell mobilization. Blood. 2003;102(4):1340-6.

16. Mueller C, Wodack K, Twelker K, Werner N, Custodis F, Nickenig G. Darbepoetin improves endothelial function and increases circulating endothelial progenitor cell number in patients with coronary artery disease. Heart. 2011;97(18):1474-8.

17. Naito T, Sanaka T, Mikami H, Soga A, Yamatani K, Segawa K, Kawashima M, Yoshikura T, Niwayama J, Nishimura H, Ogawa T, Ando A, Teramoto A, Nitta K. Modulation of circulating endothelial progenitor cells by erythropoiesis-stimulating agents in patients with chronic kidney disease stage G5 and 5D. Clin Nephrol. 2016;86(11):242-52.

18. Serizawa K, Yogo K, Tashiro Y, Kawasaki R, Endo K, Shimonaka Y, Hirata M. Epoetin beta pegol ameliorates flow-mediated dilation with improving endothelial nitric oxide synthase coupling state in nonobese diabetic rats. Cardiovasc Ther. 2017;35(2):e12250.

19. Tilling L, Hunt J, Jiang B, Sanders T, Clapp B, Chowienczyk P. Endothelial function does not relate to haemoglobin or serum erythropoietin concentrations and these do not explain the gender difference in endothelial function in healthy middle-aged men and women. Eur J Clin Invest. 2013:43(3):225-30.

20. Agarwal R. Mechanisms and mediators of hypertension induced by erythropoietin and related molecules. Nephrol Dial Transplant. 2018:33(10):1690-8
21. Rao DS, Shih MS, Mohini R. Effect of serum parathyroid hormone and bone marrow fibrosis on the response to erythropoietin in uremia. N Engl J Med. 1993;328(3):171-5

22. Petzer V, Tymoszuk P, Asshoff M, Carvalho J, Papworth J, Deantonio C, Bayliss L, Wake MS, Seifert M, Brigo N, de Souza LV, Hilbe R, Grubwieser P, Demetz E, Dichtl S, Volani C, Berger S, Boehm F, Hoffmann A, Pfeifhofer-Obermair C, von Raffay L, Sopper S, Arndt S, Bosserhoff A, Kautz L, Perrier P, Nairz M, Wolf D, Weiss G, Germaschewski V, Theurl I. A fully human anti-BMP6 antibody reduces the need for erythropoietin in rodent models of the anemia of chronic disease. Blood. 2020;136(9):1080-90.

23. Sakaguchi Y, Hamano T, Wada A, Masakane I. Types of erythropoietinstimulating agents and mortality among patients undergoing hemodialysis. J Am Soc Nephrol. 2019;30(6):1037-48.

24. Friedrich EB, Walenta K, Scharlau J, Nickenig G, Werner N. CD34-/ CD133+NEGFR-2+ endothelial progenitor cell subpopulation with potent vasoregenerative capacities. Circ Res. 2006;98(3):e20-5.

25. Yiu KH, Wang S, Mok MY, Ooi GC, Khong PL, Lau CP, Lai WH, Wong LY, Lam KF, Lau CS, Tse HF. Role of circulating endothelial progenitor cells in patients with rheumatoid arthritis with coronary calcification. J Rheumatol. 2010;37(3):529-35.

26. Gupta N, Wish JB. Hypoxia-inducible factor prolyl hydroxylase inhibitors: a potential new treatment for anemia in patients with CKD. Am J Kidney Dis. 2017;69(6):815-26.

27. Nangaku M, Hamano T, Akizawa T, Tsubakihara Y, Nagai R, Okuda N, Kurata K, Nagakubo T, Jones NP, Endo Y, Cobitz AR. Daprodustat Compared with epoetin beta pegol for anemia in Japanese patients not on dialysis: a 52-week randomized open-label phase 3 trial. Am J Nephrol. 2021;52(1):26-35.

28. Topel ML, Hayek SS, Ko YA, Sandesara PB, Tahhan AS, Hesaroieh I, Mahar E, Martin GS, Waller EK, Quyyumi AA. Sex differences in circulating progenitor cells. J Am Heart Assoc. 2017;6(10):e006245.

\section{Publisher's Note}

Springer Nature remains neutral with regard to jurisdictional claims in published maps and institutional affiliations.
Ready to submit your research? Choose BMC and benefit from:

- fast, convenient online submission

- thorough peer review by experienced researchers in your field

- rapid publication on acceptance

- support for research data, including large and complex data types

- gold Open Access which fosters wider collaboration and increased citations

- maximum visibility for your research: over $100 \mathrm{M}$ website views per year

At BMC, research is always in progress.

Learn more biomedcentral.com/submissions 